\title{
Molecular basis of 5-hydroxytryptophan synthesis in Saccharomyces cerevisiae $\dagger$
}

Cite this: Mol. BioSyst., 2016, 12,1432

Received 18th December 2015, Accepted 18th March 2016

DOI: $10.1039 / \mathrm{c} 5 \mathrm{mb} 00888 \mathrm{c}$

www.rsc.org/molecularbiosystems

We report for the first time that 5-hydroxytryptophan can be synthesized in Saccharomyces cerevisiae by heterologously expressing prokaryotic phenylalanine 4-hydroxylase or eukaryotic tryptophan 3/5-hydroxylase, together with enhanced synthesis of MH4 or BH4 cofactors. The innate DFR1 gene in the folate synthesis pathway was found to play pivotal roles in 5-hydroxytryptophan synthesis.

5-Hydroxytryptophan (5-HTP) is an intermediate in the biosynthesis of the neurotransmitter serotonin ${ }^{1}$ and has been used in the clinic for over 30 years. 5-HTP can be easily absorbed by the human body and transported across the blood-brain barrier. ${ }^{2}$ The therapeutic administration of 5-HTP has shown to be effective in treating depression as well as a wide variety of medical conditions, including fibromyalgia, insomnia, binge eating associated with obesity, cerebellar ataxia, and chronic headaches. ${ }^{3}$ Nowadays, since chemical synthesis of 5-HTP is still economically infeasible, 5-HTP is mainly extracted from the seeds of the African plant Griffonia simplicifolia, ${ }^{4-7}$ which, however, is largely limited by the season- and region-dependent supply of the raw materials.

Recently, the microbial synthesis of 5-HTP has been achieved successfully in Escherichia coli, ${ }^{6,8}$ which offers an alternative route for producing this small molecule drug. In brief, it was found that the engineered bacterial phenylalanine 4-hydroxylase $(\mathrm{P} 4 \mathrm{H})$ can effectively convert tryptophan to 5-HTP by using tetrahydromonapterin (MH4) as the cofactor. ${ }^{6,8}$ Encouraged by the pioneering studies on de novo synthesis of 5-HTP in E. coli, a model prokaryotic microorganism, in this study we aim to explore the possibility of using eukaryotic organisms such as $S$. cerevisiae to synthesize 5-HTP and uncover the molecular basis of 5-HTP synthesis in eukaryotic cells.

\footnotetext{
${ }^{a}$ Department of Biological Systems Engineering, Virginia Polytechnic Institute and State University, Blacksburg, VA 24061,USA.E-mail: xueyang@vt.edu

${ }^{b}$ Department of Pharmacology \& Toxicology, University of Arizona, Tucson, AZ 85721, USA

$\dagger$ Electronic supplementary information (ESI) available. See DOI: 10.1039/ $\mathrm{c} 5 \mathrm{mb} 00888 \mathrm{c}$

$\ddagger \mathrm{JZ}$, CW and JS contributed to this work equally.
}

Compared to $E$. coli, $S$. cerevisiae can tolerate harsher fermentation conditions and does not have a phage contamination issue. ${ }^{9}$ Such a study, to the best of our knowledge, has not yet been accomplished and little is known about the pathways and cofactors involved in 5-HTP synthesis in eukaryotic organisms. Knowing the molecular basis of 5-HTP synthesis will not only help us understand the mechanisms of 5-HTP synthesis and regulation, but also provide a promising route for large-scale production of 5 -HTP using recombinant $S$. cerevisiae as an industrial workhorse. This paper is a proof-of-concept study to reveal the molecular pathways that could lead to 5-HTP synthesis in $S$. cerevisiae. The significance of this work is that we discovered, to the best of our knowledge for the first time, that two metabolic pathways could lead to 5-HTP synthesis in $S$. cerevisiae.

To start, we surveyed some literatures and found two pathways that could potentially lead to 5-HTP synthesis in $S$. cerevisiae. The first pathway uses bacterial P4Hs for 5-HTP synthesis with MH4 used as the cofactor, ${ }^{6}$ while the second pathway uses tryptophan 5-hydroxylase (T5H) or tryptophan 3-hydroxylase (T3H) to catalyze 5-HTP synthesis from L-tryptophan by using tetrahydrobiopterin (BH4) as the cofactor ${ }^{10}$ (Fig. 1). Since it has been confirmed in E. coli that bacterial P4Hs can use MH4

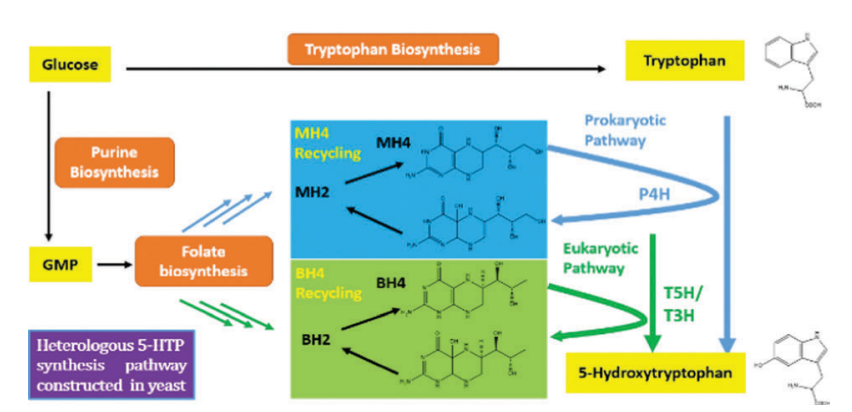

Fig. 1 Molecular basis of 5-HTP biosynthesis revealed in S. cerevisiae. Prokaryotic phenylalanine 4-hydroxylase $(\mathrm{P} 4 \mathrm{H})$ or eukaryotic tryptophan 3/5-hydroxylase (T5H/T3H) was found to synthesize 5-HTP by using $\mathrm{MH} 4$ or $\mathrm{BH} 4$ as a cofactor. 


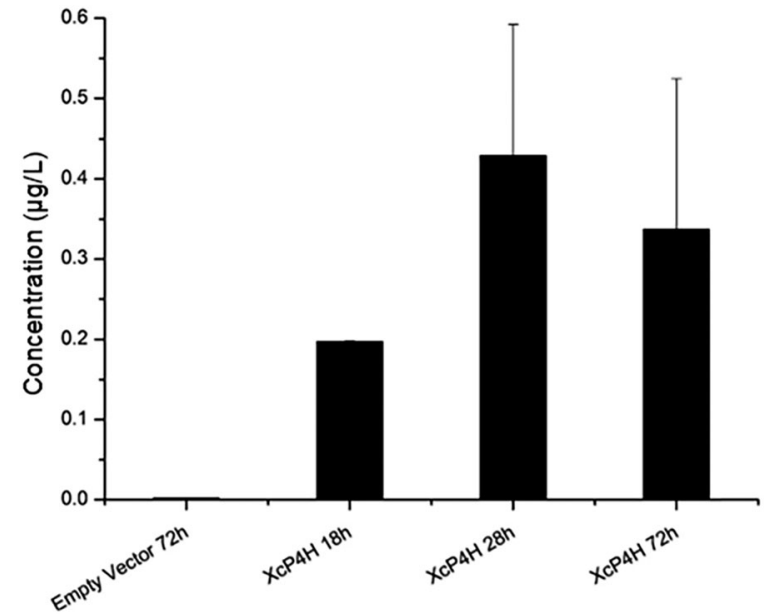

Fig. 2 5-HTP synthesis in S. cerevisiae expressing XcP4H from $X$. campestris and $\mathrm{MH} 4$ cofactor synthesis pathway. 5-HTP production was detected at $18 \mathrm{~h}, 28 \mathrm{~h}$ and $72 \mathrm{~h}$. No 5-HTP was detected in the control strain harboring an empty vector. All of the samples were measured in triplicate.

to synthesize 5-HTP, we first constructed the same bacterial pathway in $S$. cerevisiae using the DNA Assembler ${ }^{4}$ method (Tables S1 and S2, ESI $\dagger$ ). We used strong constitutive promoters to express the $\mathrm{XcP} 4 \mathrm{H}$ gene (i.e., encoding an evolved $\mathrm{P} 4 \mathrm{H}$ from $X$. campestris) and two genes for MH4 synthesis, i.e., folM gene encoding dihydromonapterin reductase (DHMR) from $E$. coli to convert dihydroxymonapetrin (MH2) to MH4; and phhB gene encoding pterin- $4 \alpha$-carbinolamine dehydratase from Pseudomonas aeruginosa to regenerate $\mathrm{MH} 2 .{ }^{6}$ We then transformed the plasmid to the $S$. cerevisiae BY4741 strain via LiAc-mediated yeast transformation, and cultured the recombinant yeast cells in SC medium with $2 \mathrm{~g} \mathrm{~L}^{-1}$ tryptophan and $1 \mathrm{mM}$ ascorbic acid in shake flasks. We measured 5-HTP concentrations (Fig. 2) in the medium at $18 \mathrm{~h}, 28 \mathrm{~h}$, and $72 \mathrm{~h}$ using an ELISA kit (Cloud-Clone Corp). As expected, we indeed detected 5-HTP produced by the recombinant $S$. cerevisiae strain, which was synthesized at $0.197 \pm 0.001 \mu \mathrm{g} \mathrm{L} \mathrm{L}^{-1}, 0.429 \pm 0.163 \mu \mathrm{g} \mathrm{\textrm {L } ^ { - 1 }}$, and $0.337 \pm$ $0.187 \mu \mathrm{g} \mathrm{L}^{-1}$ at $18 \mathrm{~h}, 28 \mathrm{~h}$, and $72 \mathrm{~h}$, respectively. Compared to the control $S$. cerevisiae strain that only expressed an empty pRS415 plasmid, the 5-HTP concentrations detected at all of the time points were significantly higher $(p<0.001)$. The decreased synthesis of 5-HTP at $72 \mathrm{~h}$ was possibly due to the degradation of 5-HTP after the long-time culture in aerobic conditions since 5-HTP is sensitive to oxidation.

Interestingly, we also found that a native $S$. cerevisiae gene, DFR1, which encodes dihydrofolate reductase to catalyze tetrahydrofolate synthesis, had a similar role as the folm gene in E. coli, by surveying the KEGG database and BioCyc database. Considering the bifurcated role of the fol $M$ gene in recycling MH4 from MH2, we hypothesized that the DFR1 gene may also have similar function for MH4 synthesis. To test our hypothesis, we constructed a series of recombinant $S$. cerevisiae strains (Table S1, ESI $\dagger$ ). As shown in Fig. 3, when we knocked out the DFR1 gene ( $\Delta d f r 1)$ and only expressed XcP4H, the 5-HTP synthesis significantly dropped $(p<0.04)$ from $2.382 \pm$ $0.255 \mu \mathrm{g}$ per L per OD to $0.520 \pm 0.235 \mu \mathrm{g}$ per L per OD because

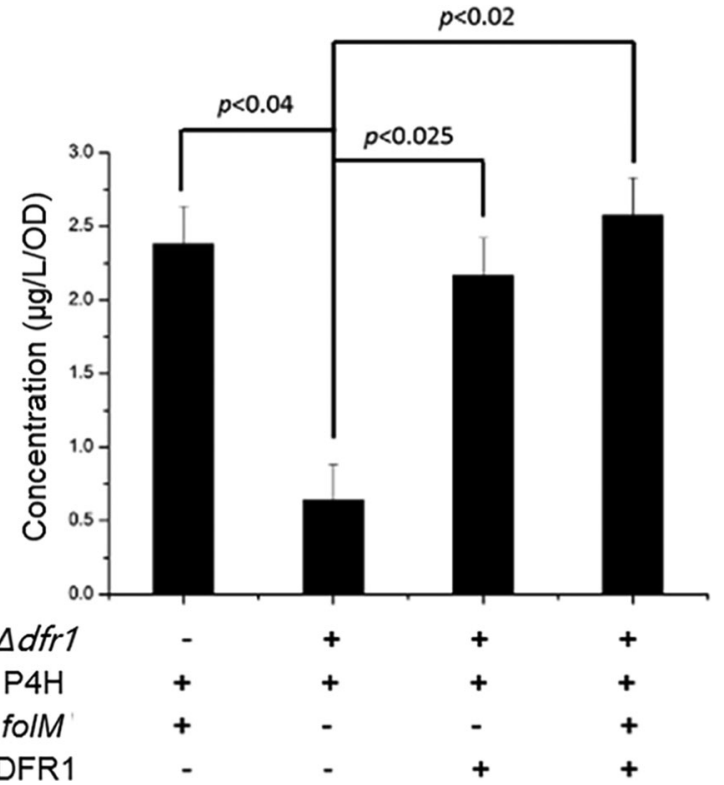

Fig. 3 The role of the DFR1 gene in 5-HTP synthesis. All of the samples were measured at $72 \mathrm{~h}$ in triplicate. Basically, we measured the concentration of 5-HTP produced in yeast as $\mu \mathrm{g} \mathrm{L}^{-1}$. In the meantime, we used the OD600 to measure the cell density. Therefore, the unit of $\mu g$ per $L$ per OD indicated the cell-based 5-HTP yields in S. cerevisiae.

of the lack of the capability to generate MH4. Then, when we co-expressed the DFR1 gene along with $\mathrm{XcP} 4 \mathrm{H}$ in the context of the forenamed $\Delta d f r 1$ knock-out mutant, the 5-HTP synthesis was fully restored, indicating that the DFR1 gene could supply MH4 for 5-HTP synthesis. When we further expressed the DFR1 gene in the $\Delta d f r 1$ mutant with expression of both $\mathrm{XcP} 4 \mathrm{H}$ and folM, the 5-HTP synthesis was slightly increased, which again suggested a positive role of the DFR1 gene in 5-HTP synthesis. Taken together, we confirmed that the DFR1 gene played a pivotal role in 5-HTP synthesis in $S$. cerevisiae by regenerating MH4. It is also worth noting that the removal of the DFR1 gene did not completely shut down the 5-HTP synthesis, indicating the presence of other innate MH4-regenerating enzymes in S. cerevisiae.

In addition to expressing bacterial P4Hs for 5-HTP synthesis with MH4 used as the cofactor, we also explored another possible pathway for 5-HTP synthesis by using eukaryotic genes. 5-HTP is natively produced in humans and animals from L-tryptophan and serves as an important hormone for humans and some animals. The synthesis of 5-HTP was catalysed by tryptophan 5-hydroxylase ( $\mathrm{T} 5 \mathrm{H})$ or tryptophan 3-hydroxylase $(\mathrm{T} 3 \mathrm{H}),{ }^{11}$ both of which use $\mathrm{BH} 4$ as the coenzyme. The $\mathrm{BH} 4$ recycling pathway was encoded by sepiapterin reductase (SPR) to catalyze the synthesis of $\mathrm{BH} 4$ from 6-pyruvoyltetrahydropterin $(6 \mathrm{PTH}),{ }^{12}$ as well as pterin-4-alpha-carbinolamine dehydratase (PCBD) and dihydropteridine reductase (DHPR) to regenerate $\mathrm{BH} 2$ and $\mathrm{BH} 4 .^{13,14}$ In addition, GTP cyclohydrolase 1 (GCH) and 6-pyruvoyltetrahydropterin synthase (PTS) could produce $6 \mathrm{PTH}$ to enhance BH4 supply. To comprehensively explore the potential for 5-HTP synthesis in S. cerevisiae, we surveyed various hydroxylases in addition to $\mathrm{XcP} 4 \mathrm{H}$, including $\mathrm{P} 4 \mathrm{H}$ from 


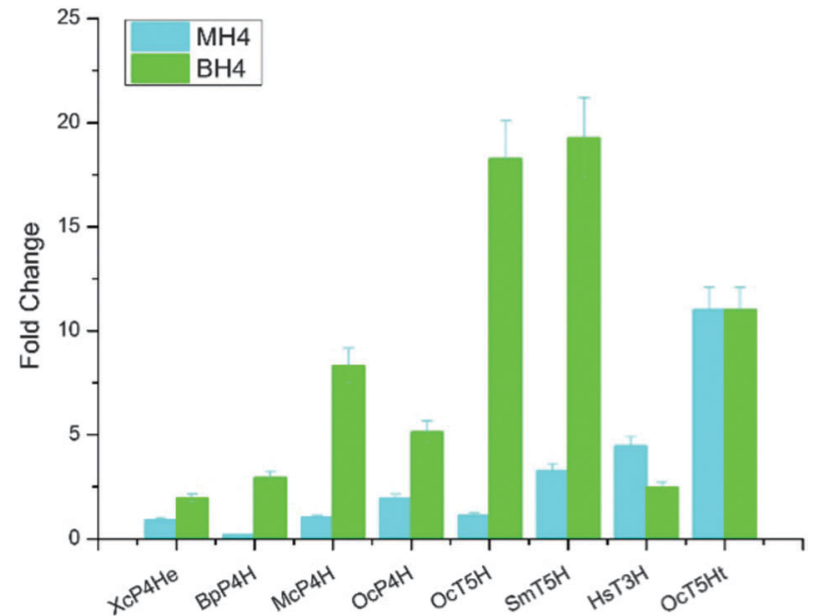

Fig. 4 5-HTP synthesis by expressing different hydroxylases (i.e., P4H, $\mathrm{T} 5 \mathrm{H}$ and $\mathrm{T} 3 \mathrm{H}$ ) and cofactor synthesis pathways (i.e., $\mathrm{MH} 4$ pathway and $\mathrm{BH} 4$ pathway). All of the samples were measured at $72 \mathrm{~h}$ in triplicate, and normalized to the $5-\mathrm{HTP}$ produced by $\mathrm{XcP} 4 \mathrm{H}$ using $\mathrm{MH} 4$ as the cofactor.

Burkholderia pseudomallei (BpP4H); $\mathrm{P} 4 \mathrm{H}$ from Mesorhizobium ciceri biovar biserrulae (McP4H); T5H from Oryctolagus cuniculu (OcT5H); a truncated version of OcT5H (OcT5Ht); T5H from Schistosoma mansoni (SmT5H); $\mathrm{P} 4 \mathrm{H}$ from Oryctolagus cuniculus (OcP4H); and $\mathrm{T} 3 \mathrm{H}$ from Homo sapiens (HsT3H). We also constructed a MH4 biosynthesis pathway by expressing folm from E. coli and phhb from P. aeruginosa; and a BH4 biosynthesis pathway by expressing PTS and SPR from Rattus norvegicus, DHPR and GCH from E. coli, and PCBD from P. aeruginosa in pRS416 plasmids of $S$. cerevisiae, respectively (Table S1, ESI $\dagger$ ). We then combined different hydroxylases with either the MH4 or BH4 biosynthesis pathway, and characterized the 5-HTP synthesis at $72 \mathrm{~h}$ by cultivating the recombinant $S$. cerevisiae strains in SC medium with $2 \mathrm{~g} \mathrm{~L}^{-1}$ tryptophan and $1 \mathrm{mM}$ ascorbic acid in shake flasks (Fig. 4). We found that OcT5H and SmT5H using $\mathrm{BH} 4$ as cofactors were the top 2 hydroxylases that led to over a 17 fold increase of 5-HTP synthesis compared to that of XcP4H using $\mathrm{MH} 4$ as cofactors. Except for HsT3H, BH4 was preferred to being used as the cofactors by all of the hydroxylases for 5-HTP synthesis in S. cerevisiae, even for those from bacteria (i.e., $\mathrm{XcP} 4 \mathrm{H}, \mathrm{BpP} 4 \mathrm{H}, \mathrm{McP} 4 \mathrm{H}$, and $\mathrm{OcP} 4 \mathrm{H}$ ). The flexibility of using either $\mathrm{MH} 4$ or $\mathrm{BH} 4$ as the cofactors might due to their similar structures and/or the low substrate specificity of different hydroxylases.

We also applied western blotting to characterize the expression levels of XcP4H, BpP4H, OcT5H, and SmT5H. These four enzymes were chosen since $\mathrm{XcP} 4 \mathrm{H}$ and $\mathrm{BpP} 4 \mathrm{H}$ led to the lowest production of 5-HTP in yeast while OcT5H and SmT5H led to the highest production. As shown in Fig. S1 (ESI $\dagger$ ), we found that the expression levels of different enzymes varied but were not positively correlated with the 5-HTP synthesis. For example, OcT5H had a similar expression level as $\mathrm{BpP} 4 \mathrm{H}$ but led to $>7$ fold higher production of 5-HTP when using $\mathrm{BH} 4$ as the cofactor. SmT5H had a higher expression level than OcT5H but led to a similar amount of 5-HTP being produced when using $\mathrm{BH} 4$ as the cofactor. In addition, we measured the in vitro enzyme activities of $\mathrm{XcP} 4 \mathrm{H}, \mathrm{BpP} 4 \mathrm{H}$, OcT5H, and SmT5H. As shown in Fig. S2 (ESI $\dagger$ ), we found that all of the enzymes demonstrated higher activities when using $\mathrm{BH} 4$ as the cofactor. The activities of OcT5H and SmT5H were 50-75\% higher than that of $\mathrm{XcP} 4 \mathrm{H}$ and $\mathrm{BpP} 4 \mathrm{H}$ when using $\mathrm{BH} 4$ as the cofactor. Taken together, the western blotting and the measurement of in vitro enzyme activities indicated that $\mathrm{T} 5 \mathrm{Hs}$ could be a better candidate when applying metabolic engineering to improve 5-HTP production in yeast.

To conclude, in this study, we discovered that two pathways could lead to 5-HTP synthesis in $S$. cerevisiae, by using either MH4 or BH4 as the cofactor and the innate DFR1 gene in S. cerevisiae was critical in recycling MH4 for 5-HTP synthesis. It is the first time, to the best of our knowledge, that the molecular basis of 5-HTP synthesis was revealed in a eukaryotic organism, which paves the ways for future metabolic engineering (e.g., increasing the supply of tryptophan precursor and cofactors) to promote yeast-based production of 5-HTP.

This work was supported by start-up fund from Virginia Tech (\#175323).

\section{Notes and references}

1 D. M. Niu, Disorders of BH4 metabolism and the treatment of patients with 6-pyruvoyl-tetrahydropterin synthase deficiency in Taiwan, Brain Dev., 2011, 33, 847-855, DOI: 10.1016/ j.braindev.2011.07.009.

2 E. A. Bell and D. H. Janzen, Medical and ecological considerations of L-dopa and 5-HTP in seeds, Nature, 1971, 229, 136-137.

3 T. C. Birdsall, 5-Hydroxytryptophan: a clinically-effective serotonin precursor, Alternative medicine review: a journal of clinical therapeutic, 1998, 3, 271-280.

4 G. Carnevale, V. Di Viesti, M. Zavatti and P. Zanoli, Anxiolytic-like effect of Griffonia simplicifolia Baill. seed extract in rats, Phytomedicine, 2011, 18, 848-851, DOI: 10.1016/j.phymed.2011.01.016.

5 P. A. Lemaire and R. K. Adosraku, An HPLC method for the direct assay of the serotonin precursor, 5-hydroxytrophan, in seeds of Griffonia simplicifolia, Phytochem. Anal., 2002, 13, 333-337, DOI: 10.1002/pca.659.

6 Y. Lin, X. Sun, Q. Yuan and Y. Yan, Engineering bacterial phenylalanine 4-hydroxylase for microbial synthesis of human neurotransmitter precursor 5-hydroxytryptophan, ACS Synth. Biol., 2014, 3, 497-505, DOI: 10.1021/sb5002505.

7 E. H. Turner, J. M. Loftis and A. D. Blackwell, Serotonin a la carte: supplementation with the serotonin precursor 5-hydroxytryptophan, Pharmacol. Ther., 2006, 109, 325-338, DOI: 10.1016/j.pharmthera.2005.06.004.

8 X. Sun, Y. Lin, Q. Yuan and Y. Yan, Precursor-Directed Biosynthesis of 5-Hydroxytryptophan Using Metabolically Engineered E. coli, ACS Synth. Biol., 2015, 4, 554-558, DOI: 10.1021/sb500303q.

9 K. K. Hong and J. Nielsen, Metabolic engineering of Saccharomyces cerevisiae: a key cell factory platform for 
future biorefineries, Cell. Mol. Life Sci., 2012, 69, 2671-2690, DOI: 10.1007/s00018-012-0945-1.

10 L. Pan, et al., Erratum: GTP-cyclohydrolase deficiency responsive to sapropterin and 5-HTP supplementation: relief of treatment-refractory depression and suicidal behaviour, BMJ, 2011, DOI: 10.1136/bcr.03.2011.3927.corr1.

11 H. Imura, Y. Nakai and T. Yoshimi, Effect of 5-hydroxytryptophan (5-HTP) on growth hormone and ACTH release in man, J. Clin. Endocrinol. Metab., 1973, 36, 204-206, DOI: 10.1210/jcem-36-1-204.
12 H. Haruki, R. Hovius, M. Gronlund Pedersen and K. Johnsson, Tetrahydrobiopterin Biosynthesis as a Potential Target of the Kynurenine Pathway Metabolite Xanthurenic Acid, J. Biol. Chem., 2016, 291, 652-657, DOI: 10.1074/jbc.C115.680488.

13 H. Myllykallio, D. Leduc, J. Filee and U. Liebl, Life without dihydrofolate reductase FolA, Trends Microbiol., 2003, 11, 220-223.

$14 \mathrm{~J}$. T. Tsay, et al., Kinetic investigation of the functional role of phenylalanine-31 of recombinant human dihydrofolate reductase, Biochemistry, 1990, 29, 6428-6436. 